\title{
Sensitivity Analysis and Stabilization for Two Dynamical Systems
}

\section{Frank Etin-Osa Bazuaye}

Department of Mathematics and Statistics, University of Port Harcourt, Port Harcourt, Rivers State, Nigeria; e-mail: febazuaye@yahoo.com

\begin{abstract}
This paper focuses on the sensitivity analysis for two dominant political parties. In contrast to Misra, Bazuaye and Khan, who developed the model without investigating the impact of varying the initial state of political parties on the solution trajectory of each political parties, we have developed a sound numerical algorithm to analyze the impact of change on the initial data on the behavior of the democratic process which is a rare contribution to knowledge. Two Matlab standard solvers for ordinary differential equations, ode 45 and ode23, have been utilized to handle these formidable mathematical problems. Our findings indicate that as the initial data varies, the dynamical system describing the interaction between two political parties is stabilized over a period of eight years. As duration increases, the systems get de-stabilized.
\end{abstract}

\section{Introduction}

The essential components of democracy are competitive elections. It is a process by which people choose an individual or group of persons to hold public offices. This process is the normal mechanism by which offices are filled in a democratic society.

The constitution and electoral act of Nigeria (2010), provides the right of individual to vote and be voted for provided he or she reaches the acceptable age. In most democratic societies, the voting system is a single vote which means voter can only vote for one candidate in the particular elective position and the candidate that receives the Received: November 18, 2018; Revised: November 28, 2018; Accepted: November 30, 2018 2010 Mathematics Subject Classification: Primary 65L05; Secondary 65L06, 65L20.

Keywords and phrases: dominant political parties, qualitative characterization, stabilization.

Copyright (C) 2019 Frank Etin-Osa Bazuaye. This is an open access article distributed under the Creative Commons Attribution License, which permits unrestricted use, distribution, and reproduction in any medium, provided the original work is properly cited. 
highest number of votes shall be declared elected by the electoral body.

It is pertinent to note that due to faulty nature and foundation of the political parties without a clear cut political ideology in these developing democracies like Nigeria, shifting or what is popularly known as "cross carpeting" of individuals from one political party to the other has become a common trend. Khan [8] conducted a research on the growth of political parties and voters. Calderon et al. [4] worked on the epidemiological approach to the spread of political third parties. However, most of the modelings use statistical approach in the analysis of the rate of shifting of political allegiance. Ekaka-a [5] carried out a novel approach to computational and mathematical modeling of plant species interactions in a harsh climate. The study of this dynamical system was not extended to the behavior of the systems in terms of the growth of two political parties. Naresh and Tripathi [7] considered using interacting models to model HIV-TB coinfection in a variable size. The non-linear differential equation with time delay was used. However, Huckfeldt and Kohfeld [6] presented two mathematical models which are linear and non-linear mathematical models in line with Morris and Pratt [9], regarding the stability of the electoral process. Bazuaye [3] considered the impact of system perturbation on the stabilization of two political parties. In the paper, the dynamical system is perturbed with different degrees of perturbation. The paper did not however address the impact of varying the initial state of the political parties on the optimal solution trajectories. This is the focus of the present study.

The present study is concerned with the numerical simulation of the impact of the initial status of any two political parties on the growth of the parties. Certainly, this approach has not been considered anywhere in literature.

\section{Mathematical Model}

Misra [1] and, Pistorius and Utterback [10] consider total population $M$ which is assumed to be constant. $M$ is divided into groups; (i) the electorates $P$, (ii) members of political party $Q$, (iii) members of political party $R$. Also, we assume that individual enters the voting class at the attainment of age 18 years and at the rate of $\varphi M$, where $\varphi$ is the rate at which individual enters or leaves the voting system, with $\varphi P, \varphi Q$ and $\varphi R$ represent those that leave the political system from each class respectively, as a result of incapacitation or death. Also, it is assumed that each electorate can either join party $Q$ or $R$ on the basis of political interest or any other personal conviction. Let $\ell_{1}$ be the average 
number of contacts of members of political party $Q$ with electorates per unit time and $\lambda_{1}$ be the probability of convincement per contact on electorate with a member of party $Q$, while the per capital recruitment rate of party $Q$ is $\eta_{1}=\ell_{1} \lambda_{1}$. So, the individual $P$ may decide to join party $Q$ at a rate $\eta_{1}(Q / M)$.

Similarly, let the per capital recruitment rate of party $R$ be $\eta_{2}=\ell_{2} \lambda_{2}$, where $\ell_{2}$ is the mean number of contacts of members of party $R$. The electorates $P$ may decide to join party $R$ at a rate $\eta_{2}(R / M)$. In addition, let $\gamma_{1}$ and $\gamma_{2}$ be the capital recruitments from party $Q$ to party $R$ and from party $R$ to party $Q$ respectively. So, members of party $Q$ leave the party at the rate $\eta_{1} Q(R / M)$ and join party $R$ at the same rate. Also, members of party $R$ leave the party at the rate $\eta_{2} R(Q / M)$ and join party $Q$ at the same rate.

Modeling equation is dynamical in nature as in Arató [2] which considered a dynamical system corresponding to the simplest Markov chain.

The Misra [1] model is given as

$$
\begin{gathered}
\frac{d P}{d t}=\varphi m-\eta_{1} P \frac{Q}{M}-\eta_{2} P \frac{R}{M}-\varphi P \\
\frac{d Q}{d t}=\eta_{1} P \frac{Q}{M}-\gamma_{1} Q \frac{R}{M}-\gamma_{2} R \frac{Q}{M}-\varphi Q \\
\frac{d R}{d t}=\eta_{2} P \frac{R}{M}+\gamma_{1} Q \frac{R}{M}-\gamma_{2} R \frac{Q}{M}-\varphi R
\end{gathered}
$$

With the initial conditions $P(0)>0, Q(0) \geq 0, R(0) \geq 0$.

At the steady state solution $\frac{d M}{d t}=0$.

\section{Method of Solution}

The numerical simulation we are proposing for the solution of this complex class of problem (2.1) is achievable with the use of mathematical ordinary differential equations solvers. The philosophy behind this method is to know the impact of varying initial data on the solution trajectory values.

Misra [1] considered the following precise deterministic parameters values:

$$
\eta_{1}=0.0417, \quad \eta_{2}=0.0278, \quad \gamma_{1}=0.0236, \quad \gamma_{2}=0.0097 \text {. }
$$


The next step is to carry out computational simulation using Matlab solvers; ode45 and ode23, to consider the impact of varying initial conditions on the solution trajectory values of the two political parties under eight years and sixteen years.

The detailed discussions and analysis are presented in the below sections.

\section{Results}

The research has shown that the stabilization of two political parties can respond to changing initial party strategy on membership drive of political parties. The stabilization is affected adversely after sixteen years as the solution trajectory values become destabilized.

\section{Discussions}

Again following Misra [1], the per capital recruitment rate $\eta_{1}$ of party $Q=0.0417$, the per capital recruitment rate $\eta_{2}$ of party $R=0.0278$.

The values of the solution trajectories are given in Tables 1 to 4 .

Table 1. Evaluating the impact of changing initial data on stabilization for 8 years simulation period using ode45.

\begin{tabular}{|c|c|c|c|c|c|}
\hline Eg & $\begin{array}{c}\text { Initial } \\
\text { condition }\end{array}$ & $\eta_{1}$ & $\eta_{2}$ & $Q_{1 e}$ & $R_{2 e}$ \\
\hline 1 & $(0.01,0.02)$ & 0.0417 & 0.0278 & 0.007557297632842 & 0.017642341756936 \\
\hline 2 & $(0.02,0.04)$ & 0.0417 & 0.0278 & 0.015050367156161 & 0.035308566937593 \\
\hline 3 & $(0.04,0.08)$ & 0.0417 & 0.0278 & 0.029845014032697 & 0.070712208514602 \\
\hline 4 & $(0.08,0.16)$ & 0.0417 & 0.0278 & 0.058676653958718 & 0.141801055013298 \\
\hline 5 & $(0.16,0.32)$ & 0.0417 & 0.0278 & 0.113375369055825 & 0.285079572992890 \\
\hline 6 & $(0.32,0.64)$ & 0.0417 & 0.0278 & 0.211435032369505 & 0.575839587279911 \\
\hline 7 & $(0.64,1.28)$ & 0.0417 & 0.0278 & 0.366228879808967 & 1.172623274761353 \\
\hline 8 & $(1.28,2.56)$ & 0.0417 & 0.0278 & 0.540229574175969 & 2.415836799751757 \\
\hline 9 & $(2.56,5.12)$ & 0.0417 & 0.0278 & 0.540132512740862 & 5.026626551929935 \\
\hline 10 & $(5.12,10.24)$ & 0.0417 & 0.0278 & 0.080303514262245 & 10.394339569501064 \\
\hline
\end{tabular}


Table 1 indicates the predicted optimal solution trajectory for a period of eight years, while $Q_{1 e}$ is the repeated simulation for the per capital recruitment rate $\eta_{1}$ of party $Q=0.0417, R_{2 e}$ is the repeated simulation for the per capital recruitment rate $\eta_{2}$ of party $R=0.0278$ using ode 45 .

Table 2. Evaluating the impact of changing initial data on stabilization for 16 years simulation period, using ode 45 .

\begin{tabular}{|c|c|c|c|c|c|}
\hline Eg & $\begin{array}{c}\text { Initial } \\
\text { condition }\end{array}$ & $\eta_{1}$ & $\eta_{2}$ & $Q_{1 e}$ & $R_{2 e}$ \\
\hline 1 & $(0.01,0.02)$ & 0.0417 & 0.0278 & 0.005396174283832 & 0.015559835168472 \\
\hline 2 & $(0.02,0.04)$ & 0.0417 & 0.0278 & 0.010682958406090 & 0.031156123337382 \\
\hline 3 & $(0.04,0.08)$ & 0.0417 & 0.0278 & 0.020932539092233 & 0.062456553875352 \\
\hline 4 & $(0.08,0.16)$ & 0.0417 & 0.0278 & 0.040164779872114 & 0.125478392115949 \\
\hline 5 & $(0.16,0.32)$ & 0.0417 & 0.0278 & 0.073789906462362 & 0.253123925099135 \\
\hline 6 & $(0.32,0.64)$ & 0.0417 & 0.0278 & 0.123442894629622 & 0.514190904780361 \\
\hline 7 & $(0.64,1.28)$ & 0.0417 & 0.0278 & 0.165290031345259 & 1.054807447746521 \\
\hline 8 & $(1.28,2.56)$ & 0.0417 & 0.0278 & 0.103291037139079 & 2.180207284657063 \\
\hline 9 & $(2.56,5.12)$ & 0.0417 & 0.0278 & -0.198941987099984 & 4.468853721493098 \\
\hline 10 & $(5.12,10.24)$ & 0.0417 & 0.0278 & -0.581965808042562 & 8.914626850183225 \\
\hline
\end{tabular}

Table 2 indicates the predicted optimal solution trajectory for a period of sixteen years, while $Q_{1 e}$ is the repeated simulation for the per capital recruitment rate $\eta_{1}$ of party $Q=0.0417, R_{2 e}$ is the repeated simulation for the per capital recruitment rate $\eta_{2}$ of party $R=0.0278$ using ode 45 .

It can be observed from Table 1 that as the first co-ordinate of the initial data ranging from 0.01 to 5.12 while the second co-ordinate is ranging from 0.02 to 10.24 , we have observed that the converging points ranging from 0.007557297632842 to 0.080303514262245 for $Q_{1 e}$ co-ordinate while the converging points for $R_{2 e}$ co-ordinate ranges from 0.015559835168472 to 10.394339569501064 under eight years. This is however different under sixteen years' duration for any political party. Table 2 shows that with the first co-ordinate of the initial data ranging from 0.01 to 5.12 , the 
second co-ordinate is ranging from 0.02 to 10.24 , we have observed that the converging points ranging from 0.005396174283832 to -0.581965808042562 for $Q_{1 e}$ co-ordinate while the converging points for $R_{2 e}$ co-ordinate ranges from 0.015559835168472 to 8.914626850183225 under sixteen years' duration.

Table 3. Evaluating the impact of changing initial data on stabilization for 8 years simulation period using ode23.

\begin{tabular}{|c|c|c|c|c|c|}
\hline Eg & $\begin{array}{c}\text { Initial } \\
\text { condition }\end{array}$ & $\eta_{1}$ & $\eta_{2}$ & $Q_{1 e}$ & $R_{2 e}$ \\
\hline 1 & $(0.01,0.02)$ & 0.0417 & 0.0278 & 0.007557297450394 & 0.017642341579574 \\
\hline 2 & $(0.02,0.04)$ & 0.0417 & 0.0278 & 0.015050366792210 & 0.035308566603801 \\
\hline 3 & $(0.04,0.08)$ & 0.0417 & 0.0278 & 0.029845013310890 & 0.070712207932630 \\
\hline 4 & $(0.08,0.16)$ & 0.0417 & 0.0278 & 0.058676652555004 & 0.141801054209269 \\
\hline 5 & $(0.16,0.32)$ & 0.0417 & 0.0278 & 0.113375366470919 & 0.285079573007056 \\
\hline 6 & $(0.32,0.64)$ & 0.0417 & 0.0278 & 0.211435027216748 & 0.575839596090450 \\
\hline 7 & $(0.64,1.28)$ & 0.0417 & 0.0278 & 0.366228828755320 & 1.172623363492501 \\
\hline 8 & $(1.28,2.56)$ & 0.0417 & 0.0278 & 0.540228141406535 & 2.415837936597692 \\
\hline 9 & $(2.56,5.12)$ & 0.0417 & 0.0278 & 0.540100422999977 & 5.026643813665331 \\
\hline 10 & $(5.12,10.24)$ & 0.0417 & 0.0278 & 0.079817020255558 & 10.394565109969625 \\
\hline
\end{tabular}

Table 3 indicates the predicted optimal solution trajectory for a period of eight years, while $Q_{1 e}$ is the repeated simulation for the per capital recruitment rate $\eta_{1}$ of party $Q=0.0417, R_{2 e}$ is the repeated simulation for the per capital recruitment rate $\eta_{2}$ of party $R=0.0278$ using ode23.

Table 4. Evaluating the impact of changing initial data on stabilization for 16 years simulation period, using ode23.

\begin{tabular}{|c|c|c|c|c|c|}
\hline Eg & $\begin{array}{c}\text { Initial } \\
\text { condition }\end{array}$ & $\eta_{1}$ & $\eta_{2}$ & $Q_{1 e}$ & $R_{2 e}$ \\
\hline 1 & $(0.01,0.02)$ & 0.0417 & 0.0278 & 0.005396171895421 & 0.0155598328366 \\
\hline 2 & $(0.02,0.04)$ & 0.0417 & 0.0278 & 0.010682953631349 & 0.03115611891808 \\
\hline 3 & $(0.04,0.08)$ & 0.0417 & 0.0278 & 0.020932529573322 & 0.06245654603760 \\
\hline
\end{tabular}




\begin{tabular}{|c|c|c|c|c|c|}
\hline 4 & $(0.08,0.16)$ & 0.0417 & 0.0278 & 0.040164761088405 & 0.12547838065901 \\
\hline 5 & $(0.16,0.32)$ & 0.0417 & 0.0278 & 0.073789870069224 & 0.2531239213151 \\
\hline 6 & $(0.32,0.64)$ & 0.0417 & 0.0278 & 0.123442808904518 & 0.5141910017180 \\
\hline 7 & $(0.64, .28)$ & 0.0417 & 0.0278 & 0.165289255329873 & 1.05480847225578 \\
\hline 8 & $(1.28,2.56)$ & 0.0417 & 0.0278 & 0.103275072728338 & 0.1802189483654 \\
\hline 9 & $(2.56,5.12)$ & 0.0417 & 0.0278 & -0.199157789853338 & 0.4689680326295 \\
\hline 10 & $(5.12,10.24)$ & 0.0417 & 0.0278 & -0.582704398819191 & 0.9149804575602 \\
\hline
\end{tabular}

Table 4 indicates the predicted optimal solution trajectory for a period of sixteen years, while $Q_{1 e}$ is the repeated simulation for the per capital recruitment rate $\eta_{1}$ of party $Q=0.0417, R_{2 e}$ is the repeated simulation for the per capital recruitment rate $\eta_{2}$ of party $R=0.0278$ using ode 23 .

The same argument holds for Table 3 and Table 4 using ode23.

It can be observed from Table 3 that as the first co-ordinate of the initial data ranging from 0.01 to 5.12 while the second co-ordinate is ranging from 0.02 to 10.24 , we have observed that the converging points ranging from 0.007557297450394 to 0.079817020255558 for $Q_{1 e}$ co-ordinate while the converging points for $R_{2 e}$ co-ordinate ranges from 0.017642341579574 to 10.394565109969625 under eight years. Also, this is different under sixteen years' duration. Table 4 shows that with the first co-ordinate of the initial data ranging from 0.01 to 5.12 , the second co-ordinate is ranging from 0.02 to 10.24 , we have observed that the converging points ranging from 0.015559832836603 to -0.582704398819191 for $Q_{1 e}$ co-ordinate while the converging points for $R_{2 e}$ co-ordinate ranges from 0.015559832836603 to 8.914980457560219 under sixteen years.

In summary, as the initial data varies, the dynamical system describing the interaction between political parties is said to be stabilized over a period of eight years. As duration increases, the system gets de-stabilized which is a true reflection of political parties in a growing democracies in the World.

Our present analysis has extended the recent contribution of Misra [1], who did not look at the impact of changing the initial data on stabilization in the context of two political parties in a developing democracy. 


\section{Conclusion}

The novel contribution of the present analysis is briefly stated as follows: By using a numerical approach, we have established the fact that the stabilization of two political parties can respond to changing initial party strategy on membership drive of political parties. This is a key contribution which has not been seen anywhere else.

\section{References}

[1] Arvind Kumar Misra, A simple mathematical model for the spread of two political parties, Nonlinear Anal. Model. Control 17(3) (2012), 343-354.

[2] M. Arató, A famous nonlinear stochastic equation (Lotka-Volterra model with diffusion), Math. Comput. Modelling 38(7/9) (2003), 709-726.

[3] Frank Etin-Osa Bazuaye, Computational simulation of the impact of system perturbation on stabilization of the growth of two political parties, J. Appl. Sci. Environ. Manage. 21(2) (2017), 391-394.

[4] K. Calderon, C. Orbe, A. Panjwani, D. M. Romero, C. Kribs-Zaleta and K. Ríos-Soto, An epidemiological approach to the spread of political third parties, mtbi.asu.edu/Summer2005.html.

[5] N. Ekaka-a, Computational and mathematical modelling of plant species interactions in a harsh climate, Ph.D. Thesis, Department of Mathematics, University of Liverpool and University of Chester, United Kingdom, 2009.

[6] R. Huckfeldt and C. W. Kohfeld, Electoral stability and the decline of class in democratic politics, Math. Comput. Modelling 16(8-9) (1992), 223-239.

[7] R. Naresh and A. Tripathi, Modeling and analysis of HIV-TB co-infection in a variable size population, Math. Model. Anal. 10(3) (2005), 275-286.

[8] Q. J. A. Khan, Hopf bifurcation in multiparty political systems with time delay in switching, Appl. Math. Lett. 13 (2000), 43-52.

[9] S. A. Morris and D. Pratt, Analysis of the Lotka-Volterra competition equations as a technological substitution model, Technological Forecasting and Social Change 70 (2003), 103-133.

[10] C. W. I. Pistorius and J. M. Utterback, Multi-mode interaction among technologies, Research Policy 26(1) (1997), 67-84. 